\title{
The effects of the observational selection criteria on the post-common envelope white dwarf-main sequence binary population
}

\author{
Judit Camacho, ${ }^{*,}$, Santiago Torres ${ }^{*, \dagger}$, Enrique García-Berro ${ }^{*,}$, Mónica \\ Zorotovic**, $^{*, \hbar}$, Matthias R. Schreiber ${ }^{\S}$ and Alberto Rebassa-Mansergas ${ }^{\S}$ \\ *Departament de Física Aplicada, Universitat Politècnica de Catalunya, c/Esteve Terrades 5, \\ 08860 Castelldefels, Spain \\ ${ }^{\dagger}$ Institut d'Estudis Espacials de Catalunya, c/Gran Capità s/n, 08034 Barcelona, Spain \\ **Departamento de Astronomía, Universidad Pontificia Católica de Chile, Chile \\ ${ }^{\ddagger}$ European Southern Observatory, Chile \\ ${ }^{\S}$ Departamento de Física y Astronomía, Universidad de Valparaíso, Chile
}

\begin{abstract}
We present a detailed Monte Carlo simulator of the Galactic population of binary stars. Preliminary results are presented for the white dwarf-main sequence (WD+MS) binary population resulting from a common envelope (CE) episode. We also study the effects of the observational selection criteria on different color-color diagrams, for different binding energy parameters, $\lambda$, and common envelope efficiencies, $\alpha_{\mathrm{CE}}$. Finally, we also compare our results with the identified population of white dwarf-main sequence binaries in the SDSS.
\end{abstract}

Keywords: stars: white dwarfs — stars: binaries — Galaxy: stellar content

PACS: 97.20.Rp, 97.10.-w, 97.80.-d, 98.35.Ln

\section{INTRODUCTION}

A wide variety of interesting astrophysical systems, ranging from low mass X-ray binaries to double degenerates or pre-CVs are formed during a common envelope phase. Although the basic ideas of common envelope evolution are nowadays generally accepted, it is still the least understood phase of evolution of close binaries. Because hydrodynamical simulations that properly follow the common envelope evolutionary phase are not currently available, the simple equations relating the total energy or angular momentum of the binary before and after the common envelope phase are generally used to predict the outcome of the system. In this work we present a Monte Carlo simulator aimed to produce a synthetic population of Galactic binaries. We place special emphasis in those systems in which one of the components of the binary system is a white dwarf and, more specifically, in WD+MS binaries. We also compare our theoretical results with the population of WD+MS binaries in the SDSS. As the evolutionary properties of both white dwarfs and main sequence stars are well understood our ultimate goal consists in understanding the evolution during the common envelope phase, thus allowing to test and calibrate the theoretical free parameters. 


\section{THE MODEL}

We have expanded an existing Monte Carlo code [1,2] specifically designed to study the Galactic populations of single white dwarfs to deal with the population of binaries in which one of the components is a white dwarf. Random zero-age main sequence (ZAMS) binaries were formed as a set of separate distributions over primary mass, mass ratio, semi-axis, eccentricities, time of birth and location. The initial primary masses $M_{1}$ were obtained using a standard initial mass function [3] and the initial mass ratios, $q=M_{2} / M_{1}$, according to a flat distribution $n(q)=q$. We only considered stars with masses $0.1 M_{\odot} \leq M \leq 30 M_{\odot}$. Also, a constant star formation rate and a disk age of $10 \mathrm{Gyr}$ were adopted. In addition, orbital separations were randomly drawn according to a logarithmic probability distribution [4]. The eccentricities were also randomly drawn according to a thermal distribution [5], $f(e)=2 e$ for $0 \leq e \leq 0.9$. For each of the components of the binary system analytical fits to detailed stellar evolutionary tracks were used $[6,7]$. The Roche lobe radius was modeled according to the standard presciption [8], and during the overflow episodes both rejuvenation and ageing were taking into account. We modeled the common envelope phase considering different prescriptions for the common envelope efficiencies and the fraction of the gravitational binding energy of the donor available to eject the envelope [9]. Specifically, we adopted $\alpha_{\mathrm{CE}}=1.0$ and $\lambda=0.50, \alpha_{\mathrm{CE}}=0.25$ and $\lambda=0.50$, and in a third set of simulations we employed $\alpha_{\mathrm{CE}}=0.25$ and variable $\lambda$ which depends on the evolutionary stage of the donor at the onset of mass transfer. The orbital evolution of the binary was computed taking into account circularization, synchronization and mass losses through stellar winds. Angular momentum losses due to magnetic braking and gravitational radiation were also taken into account $[10,11]$. Our binary systems were distributed in the Sloan Digital Survey Data Release 7 fields and we used a double exponential density law wiht a scale height $H=250 \mathrm{pc}$ and scale length $R=3.5 \mathrm{kpc}$. Also, a period filter was applied to the synthetic data. Finally, color selection criteria and spectroscopic completeness were taken into account as well.

\section{RESULTS}

Figure 1 shows the color-color diagram of present day WD+MS systems. Those systems whose progenitor underwent the $\mathrm{CE}$ phase before helium ignition (case B) are represented by black dots. Those systems whose progenitor underwent the CE episode either during the hydrogen-burning shell phase or prior to carbon ignition - corresponding to the first and second dredge-up - (case C) are represented using dark blue dots, while the systems in which the CE episode took place during the thermally pulsing phase or carbon ignition (TPAGB case) are displayed using light blue dots. Finally, red dots correspond to observational WD+MS systems. The different selection criteria are represented by red lines. Figure 2 shows the distribution of present-day WD+MS systems once cataclysmic variables were removed and all the observational biases were carefully taken into accout. As can be seen, our Monte Carlo simulator matches nicely the observed distributions of periods, white dwarf masses and secondary masses. Finally, Figure 3 shows the period histogram of the synthetic present-day WD+MS systems for our three choices of $\alpha_{\mathrm{CE}}$ 

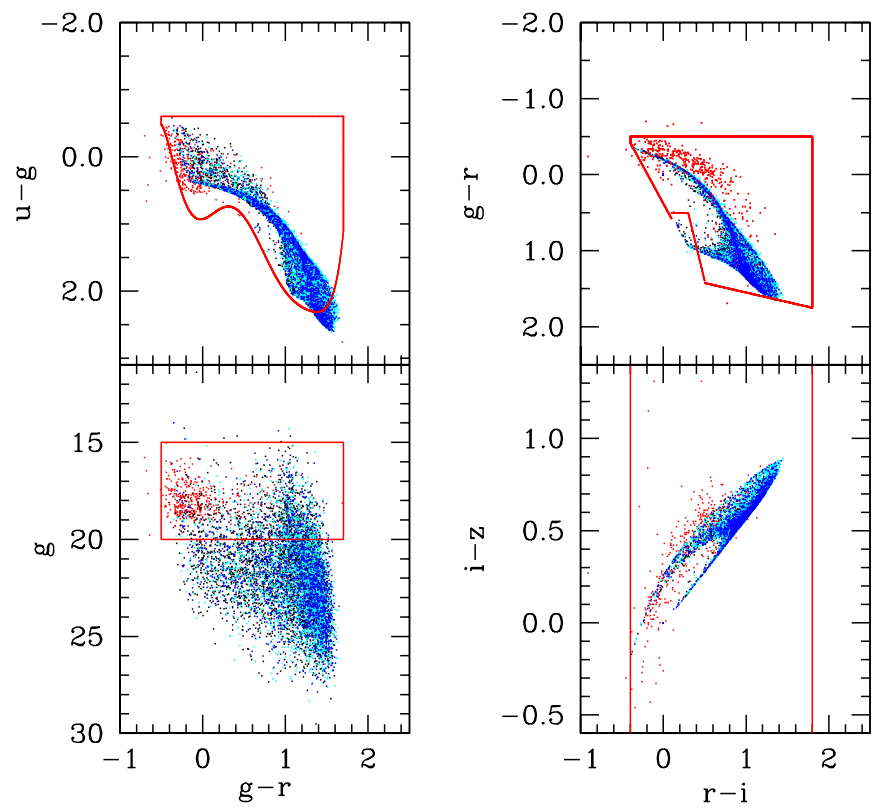

FIGURE 1. Color-color diagram of the synthetic WD+MS sytems obtained using our Monte Carlo simulator (blue and black dots) compared with the observational WD+MS systems (red dots). The different color selection criteria are shown using red lines. See text for details.
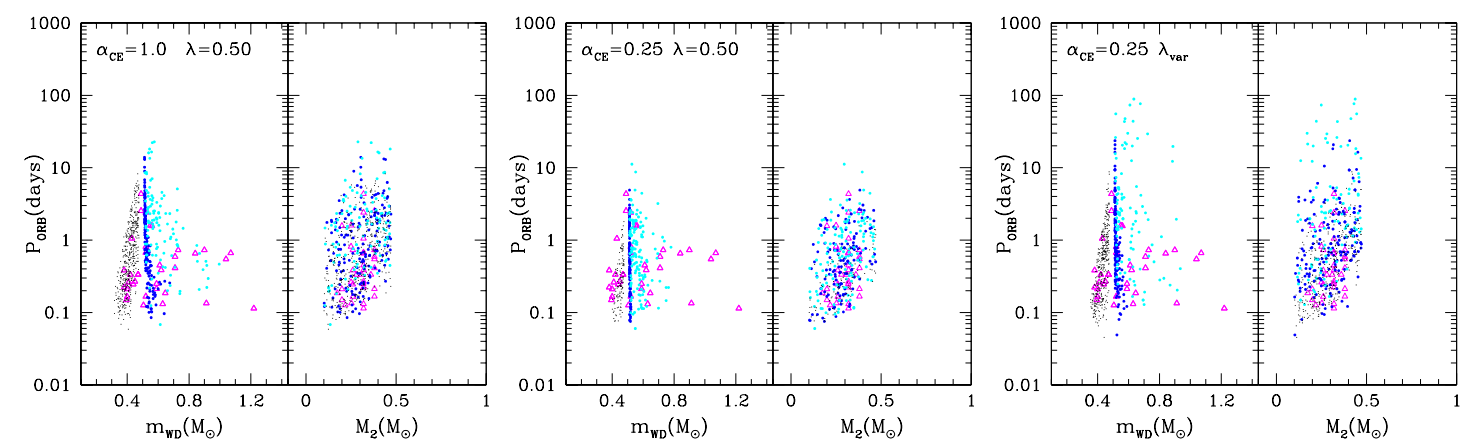

FIGURE 2. Present-day WD+MS systems once cataclysmic variables have been removed and all the observational biases have been taken into account. See text for details.

and $\lambda$. The top panels show the period histogram of case $C$ and TPAGB case binaries, while the middle panels show that of case B binaries and the bottom panels show the period histogram of the entire simulation population (black line) compared with that of the observational data (magenta line) [11]. As can be seen the agreement between our simulations and the observational data is quite good for models 2 and 3 , although a more detailed statistical analysis remains to be done. 

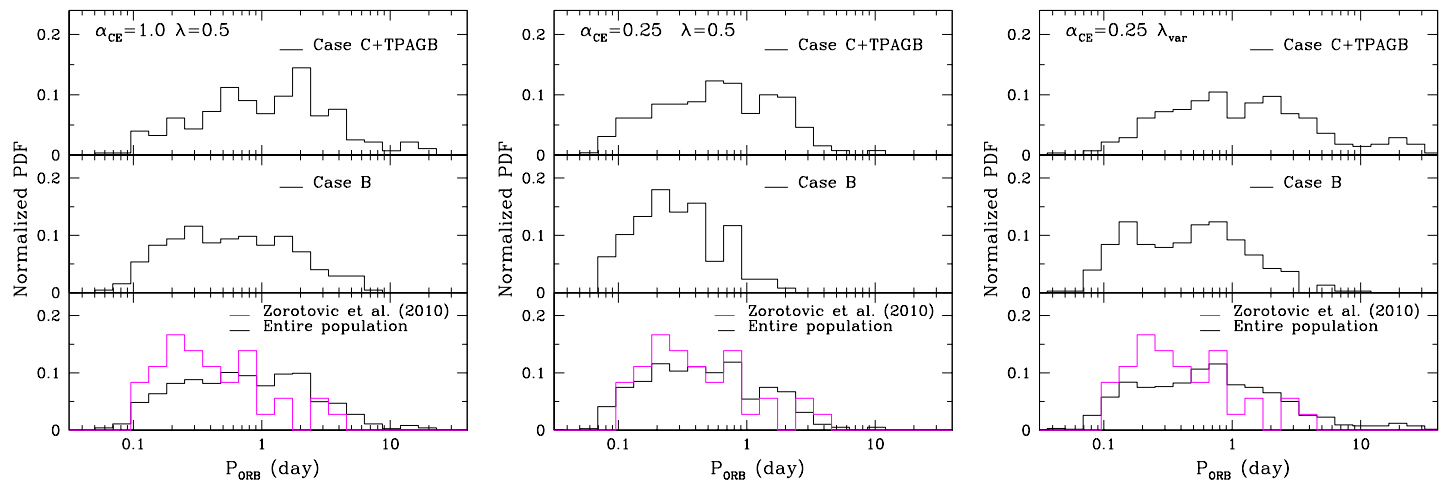

FIGURE 3. Period histograms of the distribution of present-day WD+MS systems for our three different synthetic populations (black line) compared with the observational distribution (red line).

\section{CONCLUSIONS}

We have presented a Monte Carlo simulator of the population of the post-common envelope white dwarf-main sequence binary population. We have analyzed the effects of the different observational selection criteria in the synthetic population of binaries. Additionally, we have studied the impact in the resulting populations of adopting different values of $\alpha_{\mathrm{CE}}$ and $\lambda$. Our Monte Carlo simulations correctly reproduce the observed distributions of periods, white dwarfs and secondary masses. In particular, models 2 and 3 are those which best match the observational distributions of orbital periods and, specifically, models with a variable $\lambda$ best fit the observational distribution for large periods.

\section{ACKNOWLEDGMENTS}

This work was supported by MEC grant AYA08-04211-C02-01, by AECID grant A/023687/09 and by the AGAUR.

\section{REFERENCES}

1. E. García-Berro, S. Torres, J. Isern, and A. Burkert, Month. Not. Roy. Astron. Soc. 302, 173-188 (1999).

2. E. García-Berro, S. Torres, J. Isern, and A. Burkert, Astron. \& Astrophys. 418, 53-65 (2004).

3. P. Kroupa, C. A. Tout, and G. Gilmore, Month. Not. Roy. Astron. Soc. 262, 545-587 (1993).

4. G. Nelemans, L. R. Yungelson, S. F. Portegies Zwart, and F. Verbunt, Astron. \& Astrophys. 365, 491-507 (2001).

5. D. C. Heggie, Month. Not. Roy. Astron. Soc. 173, 729-787 (1975).

6. J. R. Hurley, O. R. Pols, and C. A. Tout, Month. Not. Roy. Astron. Soc. 315, 543-569 (2000).

7. I. Renedo, L. G. Althaus, M. M. Miller Bertolami, A. D. Romero, A. H. Córsico, R. D. Rohrmann, and E. García-Berro, Astrophys. J. 717, 183-195 (2010).

8. P. P. Eggleton, Astrophys. J. 268, 368-+ (1983).

9. M. de Kool, Astrophys. J. 358, 189-195 (1990).

10. M. R. Schreiber, and B. T. Gänsicke, Astron. \& Astrophys. 406, 305-321 (2003).

11. M. Zorotovic, M. R. Schreiber, B. T. Gänsicke, and A. Nebot Gómez-Morán, ArXiv e-prints (2010), 1006.1621 . 